\title{
El reto de la soledad en la vejez
}

\section{Javier Yanguas Lezaun}

Director Científico del Programa de Mayores de la Fundación Bancaria "la Caixa" y presidente de la Sección de Ciencias Sociales y del Comportamiento de la International Association of Gerontology and Geriatrics for the European Region

\section{Amaya Cilveti Sarasola}

Coordinadora del programa "Siempre Acompañados" de la

Fundación Bancaria "la Caixa"

acilveti@fundaciolacaixa.org

\section{Sonia Hernández Chamorro}

Coordinadora del programa "Sempre Acompanyats" de Creu Roja a Catalunya

\section{Sacramento Pinazo-Hernandis}

Vicepresidenta de Gerontología de la Sociedad Española de Geriatría y Gerontología y profesora titular de Psicología Social de la Universidad de Valencia

\section{Susanna Roig i Canals}

Directora de Intervención Social de Creu Roja a Catalunya

\section{Cristina Segura Talavera}

Directora del Programa de Mayores de la Fundación Bancaria “la Caixa”

Artikulu honetan, pertsonen arteko harremanen garrantzitik abiatuz, zahartzaroko bakardadearen fenomenora hurbiltzen gara. Jarraian, bakardadeak duen konplexutasuna eta bai osasun fisikoan nola osasun psikologikoan duen eragina eztabaidatzen da gaur egun ditugun datuak emanez. Horretaz gain, alde batetik interbentzioen eraginkortasuna aztertzen du artikuluak, eta bestetik, "Siempre Acompañados" izeneko programa eta burutu den hasierako froga.

\section{GAKO-HITZAK:}

Bakardadea, zahartzaroa, prebentzioa, giza harremanak.
En el presente artículo se aborda el fenómeno de la soledad en personas mayores partiendo de la importancia del funcionamiento social y las relaciones sociales en el envejecimiento. Seguidamente se expone una visión multidimensional del complejo fenómeno que es la soledad en la vejez; abordando tanto las cifras relacionadas con el reto que supone, como sus consecuencias en la salud física y psicológica. El artículo recoge también un análisis de la evidencia empírica existente en torno a intervenciones para la prevención de la soledad, profundizando en la conceptualización, descripción y principales aprendizajes derivados del estudio piloto efectuado dentro del programa "Siempre Acompañados" para disminuir la soledad no deseada entre las personas mayores.

\section{PALABRAS Clave:}

Soledad, envejecimiento, prevención, relaciones sociales. 


\section{Introducción}

El profesor Robert Weiss, uno de los pioneros en el estudio de la soledad, les proponía a sus alumnos en los ochenta del siglo pasado en los Estados Unidos el siguiente ejercicio:

“Por favor, cierra los ojos.

Vives en un apartamento. Estás sola. Por lo que se refiere a tus sentimientos, estás completamente sola. No tienes a nadie a quien llamar, a nadie con quien hablar. Nadie comparte tu vida, nadie en absoluto. Así es como están las cosas, y así es como van a seguir.

Si salieras a la calle, seguirías estando sola.

Por favor, toma nota mental de cómo te sientes. Ahora abre los ojos y anota cuáles son tus sentimientos".

\section{Envejecimiento y relaciones sociales}

La importancia de las relaciones sociales en la vejez, así como su influencia en la salud y el bienestar, ha sido ampliamente estudiada desde hace décadas (Cumming y Henry, 1961; Kahn y Antonucci, 1980). Durante todo el ciclo vital se producen cambios asociados al hecho de envejecer -e independientes del fenómeno de la soledad-en los tres conceptos esenciales a través de los cuales se describen las relaciones sociales: la red social, el apoyo social y la participación social (Holt-Lunstad et al., 2010).

Las redes sociales se pueden definir como el elemento estructural de las relaciones sociales. Habitualmente se ha hecho énfasis en el tamaño y la frecuencia de los contactos, pero revisando algunas de las pruebas que la evalúan - por ejemplo, la de red social de Lubben (Lubben, 1988; Lubben et al., 2016) aunque no únicamente-, estas suelen abordan los tres aspectos esenciales que conceptualiza la red social: el emocional, la "tangibilidad" y el tamaño.

El apoyo social, en cambio, es un proceso transaccional (dar y recibir), a través del cual nuestras relaciones nos proporcionan un "espacio" para el intercambio tanto de experiencias emocionales como de apoyo en cuestiones instrumentales cotidianas. Se suele clasificar según la función del apoyo: emocional, material o instrumental e informacional; o el tipo, formal (profesional), informal (no profesional) y familiar.

Por último, la participación social (“engagement”) se relaciona con la frecuencia y la calidad de las actividades formales (por ejemplo, actividades religiosas, asistencia a reuniones, ser voluntario, etc.) e informales (por ejemplo, contactos telefónicos, quedar con amigos, etc.) que una persona realiza con los miembros de su red social.
A lo largo del ciclo vital se van dando cambios en nuestro funcionamiento social y más concretamente en la vejez que se caracterizan por:

- La evidencia empírica demuestra que existe una reducción de los distintos parámetros relacionados con la red social, asociados al hecho de envejecer, que no debemos confundir con la soledad. La edad está asociada con redes sociales más pequeñas y menor frecuencia de contactos (Ajrouch et al., 2005), particularmente en el caso de los hombres mayores (Cornwell et al., 2008); siendo las relaciones sociales que no son de parentesco menos comunes entre los mayores, que dirigen hacia los miembros de su familia su mundo relacional (Pahl y Pevalin, 2005).

- Respecto a la calidad de las relaciones, no parece que exista afectación a lo largo del proceso de envejecimiento: las personas mayores tienen relaciones de mayor calidad dentro de esa red social más pequeña y alcanzan mayores niveles de participación social en su comunidad que otras personas de menor edad (Carstensen, 1992) en ausencia de situaciones de dependencia.

- Los mayores, en contra de lo que a veces estereotipadamente se afirma con rotundidad, tienen mayores contactos con vecinos y es más probable que sean voluntarios (salvaguardada la salud) que personas de menor edad (Cornwell et al., 2008).

- Para las personas mayores, la familia -en particular el cónyuge y los hijos adultos- son la fuente central de apoyo (Waite y Gallagher, 2000), teniendo el matrimonio (y el hecho de vivir en pareja) un efecto protector tanto en la salud física (Waite y Gallagher, 2000) como en el bienestar psicológico (Schwarzbach et al., 2014).

- En el envejecimiento, los amigos siguen desempeñando un papel fundamental: a través de estudios longitudinales (Cable et al., 2013) se ha comprobado que el tamaño de la red de amigos se relaciona con una mejor salud psicológica. Se puede afirmar que cuanto más variada es la red social, más felices y sanas son las personas en la vejez; en otras palabras, los amigos son esenciales para los mayores, especialmente en ausencia de familia (cuestión que, vista la demografía, será cada vez más común).

Desde la gerontología se ha intentado explicar estos cambios en las relaciones sociales en la vejez, más allá de constatar su reducción a medida que las personas sobreviven a sus amigos. Hay tres teorías esenciales:

- La primera fue la teoría de la desvinculación de Cumming y Henry (1961), según la cual los mayores se retiran activamente de su red y reducen el número de amigos como resultado de la disminución de su salud física, pérdida de roles, preparación de la propia muerte, etc.

- La teoría de la selectividad socioemocional (Carstensen, 1992) ofrece un punto de vista 
alternativo al anterior, donde en la medida que envejecemos nos volvemos más selectivos y fortalecemos los lazos emocionales de mayor calidad, disolviendo lo periférico y creando (a costa del tamaño) menor número de relaciones pero de más alta calidad, porque lo sustancial en la vejez son los aspectos emocionales de las relaciones.

- Un tercer modelo, llamado de "convoy social" (Kahn y Antonucci, 1980), describe patrones de cambio en las relaciones sociales a lo largo del envejecimiento según los cambios que se producen tanto a nivel personal (debido a la edad, al género, o en el estado de salud, etc.), como a factores situacionales (roles, valores, cultura, etc.), y cómo dichos cambios afectan a la cantidad y calidad de relaciones, sugiriendo el modelo que nuestro "convoy" de relaciones cambia a medida que envejecemos, poniendo de relieve la creciente importancia de la calidad emocional respecto a la cantidad de relaciones a lo largo del proceso de envejecimiento.

En los últimos decenios, la investigación ha demostrado la influencia de las relaciones sociales en la salud (Umberson y Montez, 2010) y en la mortalidad: las personas con buenas relaciones sociales (incluyendo tanto desde el punto de vista funcional como estructural) tienen un riesgo de mortalidad un cincuenta por ciento menor en comparación con los que declaran ser pobres relaciones sociales (Holt-Lunstad et al., 2010).

¿Qué sucede con las relaciones sociales a lo largo del proceso de envejecimiento? Básicamente que existe una disminución de la "cantidad" de todos los parámetros relacionados con el funcionamiento social, en un proceso "natural" (propio del ciclo vital) a través del que se busca mejorar la "calidad" de esos contactos y con ello maximizar el bienestar (uno de los grandes objetivos de los cambios durante el ciclo vital). Conjuntamente con este proceso de cambio en el funcionamiento social, en la vejez existe un conjunto de "situaciones" (enfermedades, fragilidad, pérdidas, dependencia, necesidad de creación de un nuevo proyecto vital, jubilación, cambios en la familia, etc.) que son más comunes (estadísticamente hablando) y que hacen que sea más probable que desaparezcan esas redes de apoyo, y aumentan los sentimientos de soledad y desvinculación. Así como los cambios para maximizar el bienestar son "propios" del ciclo vital, la soledad es un fenómeno "no buscado", más probable quizá, pero no "característico" o "idiosincrático" de las etapas final de la vida.

Una última cuestión: las relaciones sociales funcionan como "amortiguadores del estrés", es decir, otras personas interfieren entre "la persona" y las "dificultades" a las que se enfrenta. Nuestras relaciones sociales pueden ofrecernos apoyo (emocional, informacional, instrumental), pueden ayudarnos a reducir los efectos que un determinado problema o situación tienen en nuestra vida. En otras palabras: cuando tenemos un problema, recurrimos a nuestras fuentes de apoyo. Las personas en situación de soledad carecen de este preciado recurso.

Sencillamente esto no se da. Ante cualquier situación no tienen a nadie a quien recurrir. En el hipotético caso de que tengan apoyo instrumental (alguien que les ayude en alguna tarea concreta), es muy probable que no sientan el apoyo psicológico, porque se sienten solos. No existe nadie que les refuerce positivamente. Y esta falta de apoyo, además del aumento del sufrimiento, parece fundamental en la aparición de los problemas de salud que más adelante se tratarán. Cuando más se necesita a las personas, más se abre un abismo entre la necesidad de los otros y las posibilidades que la persona (con soledad) cree tener de poder estar con otros.

\section{Definiendo la soledad}

Hasta hace aproximadamente medio siglo, la soledad había sido objeto exclusivo de las artes y la filosofía, y había tenido un escaso eco de las ciencias (Weiss et al., 1973a; Weiss et al, 1973b; Weiss, 1983; Sønderby, 2013). Es en 1982 cuando se publica el primer manual sobre soledad, titulado Loneliness: a sourcebook of current theory, research and therapy, en el que uno de sus intereses fue conceptualizar la soledad desde su riqueza y complejidad, categorizando ocho distintas aproximaciones teóricas a la soledad (p. 130) resumibles en cuatro perspectivas clásicas (Yanguas et al., 2018):

1. La perspectiva cognitiva, en la que la soledad se entiende como una discrepancia (una evaluación que el sujeto realiza) entre las relaciones sociales que una persona desea y las que tiene.

2. El interaccionismo, que conceptualiza la soledad no desde el hecho de estar solo, sino desde la falta de relaciones tanto significativas como íntimas, así como por la falta de vinculación comunitaria.

3. La perspectiva psicodinámica, que concibe la soledad como las consecuencias negativas derivadas de la necesidad de intimidad, de relaciones interpersonales para vivir.

4. El existencialismo, para el que la soledad es una realidad consustancial al ser humano que, por un lado, puede causar sufrimiento y dolor, y por otro, supone la posibilidad de crear cosas nuevas, reflexionar y comprenderse a uno mismo, etc.

Dieciséis años más tarde, en 1989, se publica un segundo manual titulado Loneliness: Theory, Research and Applications (Hojat y Crandall, 2009) donde se aprecia que el interés ha basculado desde las distintas teorías que diferenciaban qué es y cómo se puede comprender la soledad (una mirada más rica sobre ella), hasta teorías de corte más empírico, que enfatizan la importancia de medir la soledad, donde se asume básicamente que existe en ella: 
un componente afectivo, ligado a las emociones negativas (y sustentado por una aproximación teórica de corte psicodinámico que enfatiza la importancia de la necesidad social de relación); y un componente cognitivo (que es el que en realidad adquiere importancia en la visión de la soledad), relacionado con la discrepancia entre expectativas y realidad propia de las visiones cognitivistas.

Durante estos últimos decenios, distintos autores han intentado definir la soledad, aprehender el concepto, algunos reduciéndolo sin piedad a cuestiones superficiales (por ejemplo, aquellos que comparan soledad con aislamiento). Rubenstein y Shaver $(1980,1982)$ ya nos avisaban de dicho peligro cuando nos decían refiriéndose a la soledad que "la psicología ha demostrado otra vez su notable capacidad para reducir las profundas y fascinantes cuestiones humanas a generalizaciones más bien superficiales y poco interesantes", y otros buscando una mirada más profunda y poliédrica. Resumidamente, y con la esperanza de no ofender a Rubenstein y Shaver, la soledad tiene muy diversos abordajes no mutuamente excluyentes, que deben ser comprendidos y conceptualizados desde la integración de diversas perspectivas complementarias. Así, la soledad es:

- Un sentimiento de aislamiento, que puede ser objetivo o subjetivo, metafísico o comunicativo, existencial, social, etc., que está siempre presente y que es parte nuclear de la experiencia personal e intransferible de lo que llamamos soledad (Stein y Tuval-Mashiach, 2015).

- Puede ser también carencia o privación referida a sentimientos de vacío o abandono asociados a la ausencia de relaciones de intimidad (De Jong Gierveld, 1987), en otras palabras, la soledad tiene en su génesis un fuerte componente relacional.

- Es fruto a la vez de una discrepancia cognitiva entre las relaciones que una persona tiene y las que esperaba tener, cuya aparición y mantenimiento dependen de la evaluación subjetiva de la propia persona sobre la calidad y la cantidad de sus relaciones sociales (Peplau y Perlman, 1982; Yanguas et al., 2018).

- Incluye siempre un conjunto de aspectos emocionales que la acompañan, como tristeza, melancolía, frustración, vergüenza o desesperación (De Jong Gierveld, 1987; De Jong Gierveld et al., 2015; Yanguas et al., 2018; Pinazo y Donio Bellegarde, 2018), habitualmente ligados con la presencia de un déficit, que siempre conlleva amargura y dolor (aunque a través de ella una persona -hipotéticamente- pueda aprender, mejorar, desarrollarse personalmente, etc.).

- Puede derivarse de causas objetivas (ausencia de relaciones, por ejemplo) o ser independiente de ellas (una persona puede no estar solo, pero sentirse solo), no estando obligatoriamente relacionada con las habilidades sociales del sujeto que la padece (Vitkus y Horowitz, 1987): ante la creencia de que los solitarios tienen malas relaciones sociales, la evidencia empírica avisa de que muchas personas en situación de soledad tienen buenas habilidades sociales.

- Además de poder venir provocada por una falta de relación con otras personas, también puede tener un componente de falta de vinculación comunitaria. La comunidad donde vive un individuo le provee de sentimientos de pertenencia, identificación con otros, seguridad emocional, influencia recíproca, percepción de compartir valores y recursos, conexión emocional además de satisfacción de necesidades, etc., cuya ausencia puede provocar sentimientos de soledad (Dalton, Elias y Wandersman, 2001). En este sentido, autores relevantes como Cacioppo y Patrick (2008) definen la soledad como un "dolor social" comparable al dolor físico, dotándola de una función específica: si el dolor físico surge para protegernos de los peligros físicos, la soledad, como "dolor social", se manifestaría como una forma de protegernos del peligro de permanecer aislados (relacionado, obviamente, con la importancia de las conexiones sociales).

- Necesita un tiempo para fraguarse (una persona no siente soledad, aunque se pueda sentir solo, de un día para otro), requiere, por lo tanto, de una perspectiva temporal a través de la cual el individuo construye y percibe su soledad (De Jong Gierveld, 1998).

- Es una experiencia vivida en forma de múltiples realidades (Victor et al., 2009; Victor y Sullivan, 2015), que son experiencias personales únicas, distintas y cambiantes que el individuo construye y reconstruye en el contexto de su vida e historia de vida, cuyos matices cambian a lo largo del tiempo. La soledad es algo dinámico y exige intervenciones capaces de asumir eso.

- Existen tantas soledades como "porqués" generadores de los distintos sentimientos de aislamiento, vacío, exclusión, etc., que experimenta una persona en situación de soledad (Yanguas, 2018).

- La soledad depende de la interacción mutua de diversas variables, algunas propias de la persona y otras externas al individuo (algunas bajo su influencia y otras que no dependen en absoluto de su voluntad) en mutua interacción (Victor et al., 2009; Victor y Sullivan 2016):

- Factores intrapersonales: personalidad y "estilos" cognitivos (expectativas, evaluación de la situación por parte de la persona, etc.).

- Factores extrapersonales, como el "engagement interpersonal” (es decir, el funcionamiento social de la persona a lo largo de todo su ciclo vital); los eventos vitales que les suceden a las personas tanto relativos a su salud (enfermedades, etc.) como sociales (jubilación, viudez, pérdidas, marcha de los hijos a otros países, etc.); factores socioeconómicos (renta, existencia 
o no de servicios de atención; el "ambiente social” en el que viven (vivienda, barreras arquitectónicas, equipamientos, tipo de comunidad más individualista o colectiva, ámbito rural versus urbano), etc.; estilos de vida (uso del tiempo libre, aficiones, etc.); factores culturales, estereotipos sociales (edadismo), etc.

- La soledad es vista habitualmente de manera opuesta por los que la padecen y por lo que no la experimentan (Hauge y Kirkevold, 2010; Donio Bellegarde, 2017): los "no solos" suelen opinar que la culpa de la soledad corresponde a la propia persona solitaria, siendo causada, por ejemplo, por su personalidad crítica, por su actitud pasiva y negativa, etc., haciendo una atribución causal interna; los "solos" generalmente la atribuyen más a factores externos, por ejemplo a la falta de oportunidades de mantener el contacto social con personas relevantes, que a algo provocado por ellos mismos, es decir, hacen una atribución causal externa.

- Robert Weiss (1983), uno de los pioneros en el estudio de la soledad, avisaba hace más de treinta años de la importancia de los estereotipos nocivos que la soledad tenía. Según este autor, la soledad es vista a menudo como debilidad, como flaqueza o como autocomplacencia, ya que se supone que todo individuo debería ser capaz de eliminarla, al no tratarse de una dolencia de tipo físico. Como sucede con la tristeza o la depresión, continuaba Weiss, parecería que el sentimiento de soledad pudiera ser eliminado exclusivamente a través de la voluntad, y que el individuo en último término aparece culpable de ella, bien por acción u omisión. Estos estereotipos, desgraciadamente, siguen hoy bien presentes como constata la evidencia empírica existente, tanto en población general (Sagan y Miller, 2017), como en personas mayores (Pikhartova, Bowling y Victor, 2016) subrayándose tanto un comportamiento diferente hacia las personas supuestamente solitarias (más frio y distante), como de profecías autocumplidas respecto a la soledad en mayores.

- Rubestein y Shaver $(1980,1982)$ contemplaban la complejidad de la soledad (y anunciaban como debería la respuesta a ella) a través de taxonomías donde diferenciaban los distintos sentimientos de soledad, los motivos que hipotéticamente los podían ocasionar y las respuestas de las personas ante dichos sentimientos de soledad. Así:

- Dentro de los distintos sentimientos, argumentaban que existe un tipo de soledad ligada a los sentimientos de desesperación (que incluyen miedo, desesperanza, vulnerabilidad, etc.); otra categoría de soledad provocada por sentimientos de tristeza, vacío, aislamiento, etc.; otra relacionada con el hastío, el aburrimiento, etc.; otra con la baja autoestima; etc.
- Los sentimientos que caracterizan las distintas soledades son producidos por causas distintas: hay quien se puede sentir solo porque se siente aislado, o porque le falta la pareja; o, teniendo pareja, no cuenta con el cariño que necesita; o porque está hospitalizado, o por miles de posibles causas distintas. La cuestión que cabe plantearse es: ¿ante motivos diferentes, dar compañía es suficiente? 0, ¿si queremos paliar, aliviar o mejorar la soledad solo podemos hacerlo mediante un conjunto de intervenciones múltiples y variadas?

- Ante sentimientos diversos y causas diferentes que provocan las distintas soledades, los individuos reaccionan de formas muy variadas. Así, en la soledad nos encontramos por un lado con una enorme diversidad de respuestas personales a su situación de soledad (hay quien elabora una respuesta activa, otros pasiva, hay quien busca apoyo social, etc.); por otro, con la importancia de las capacidades de las personas dando respuestas a la soledad.

- Diversas investigaciones han relacionado la soledad actual de un individuo con su trayectoria vital (Aarstsen y Jylhä, 2011). La participación social en las personas mayores está determinada por la que un individuo experimenta al menos veinte años antes, y hay una relación directa entre la participación social y la soledad (Dahlberg et al., 2016). En otras palabras, la soledad no es un producto exclusivo del momento actual, sino de la historia relacional de cada uno.

Así pues, el fenómeno de la soledad, lejos de ser algo sencillo, comprende desde emociones hasta procesos cognitivos, incluye a la persona y a la comunidad, engloba variables intrapersonales y culturales, se ve influencia por los comportamientos y mediatizada por factores externos como la vivienda, y se relaciona con fragilidad y vulnerabilidad y un largo etcétera de cuestiones en las cuales residen tanto su complejidad como el desafío a nuestras sociedades.

\section{La soledad en datos}

Habitualmente se suelen dar datos de prevalencia de la soledad, confundiéndose "soledad" con "vivir solo". Siguiendo esta analogía, en España en el 2015 , el $25 \%$ de la población vivía sola, alrededor de 4.585.200 personas, según datos del Instituto Nacional de Estadística (INE). De ese conjunto total de hogares donde viven personas solas, en el $40 \%$ viven personas mayores de 65 años. De ellas, 7 de cada 10 viven solas y son mujeres; $y$ aproximadamente habría casi 400.000 mayores de 85 años, en la inmensa mayoría de los casos mujeres, que viven solas (INE, 2015). Los datos del 2017 (INE, 2017) confirman que la tendencia a vivir solo cuando se es mayor se acentúa: de las 
4.687.400 personas que vivían solas en el 2017, un 41,8 \% (casi 2 millones) tenían 65 o más años y, de ellas, 1.410.000 eran mujeres. En Euskadi, en el 2017, 247.700 personas viven solas; de ellas, 103.800 (un $41,9 \%$ ) tienen 65 o más años y 76.400 son mujeres. Resumiendo: cada vez más personas mayores y no mayores viven solas. Esto, que puede ser considerado tanto un "triunfo social" (antes no era posible), como "ley de vida", que puede ser deseado o impuesto, es también un riesgo y puede tener consecuencias, más si cabe cuando uno es mayor.

No obstante, esta analogía no es absolutamente cierta. El aislamiento social se refiere a la falta de contacto social, que pueden cuantificarse objetivamente (por ejemplo, viviendo solo). La soledad, sin embargo, se define subjetivamente como una experiencia dolorosa experimentada en ausencia de relaciones sociales, de sentimientos de pertenencia o debida a una sensación de aislamiento (Mushtaq et al., 2014; Hawkley y Capitanio, 2015). La soledad, como se ha mencionado en el punto anterior, es también fruto de una discrepancia percibida entre las necesidades sociales y su disponibilidad; es, como destacaba Weis (1987), un estado emocional que refleja la experiencia subjetiva del que sufre aislamiento social. Por ello, aunque exista una intensa relación entre "vivir solo" y "sentirse solo", el análisis de la soledad no solo es factible hacerlo únicamente desde los "modos de convivencia", sino fundamentalmente analizando dos cuestiones: la red social y los sentimientos de soledad.

Los estudios que abordan la soledad, desde el punto de vista de la red social (el aislamiento social) y los sentimientos de soledad, dan una imagen brumosa, poco nítida en algunos casos, con datos muy dispares e incluso contradictorios, con muestras habitualmente de otras culturas (la soledad, como veremos está muy ligada a factores culturales) y a veces difícilmente comparables por cuestiones metodológicas (diseño de la muestra, pruebas de evaluación, distintas formas de preguntar por la soledad, etc.). Asumiendo lo anteriormente reseñado, una foto del reto que la soledad supone a las sociedades occidentales sería la siguiente:

- Según datos de Eurostat (2017), el $6 \%$ de la población de la Unión Europea (UE) no tiene a nadie a quien pedir ayuda si la necesita (muestra de adultos de 16 años o más), con datos que varían entre el $2 \%$ de países como la República Checa, Finlandia, Eslovaquia y Suecia, y el $13 \%$ de Italia y Luxemburgo. Un porcentaje similar de la población de la UE, el $6 \%$, no tiene a nadie con quien tratar o discutir sus asuntos personales. En este sentido, las cifras más altas se registraron en Francia e Italia (12\%) y las más bajas en Chipre, España, Eslovaquia, República Checa y Hungría (2\%). Además, estos datos destacan otra cuestión: las desigualdades socioeconómicas parecen ser fundamentales a la hora de tratar el aislamiento social, ya que la diferencia de la tasa de aislamiento social entre los europeos con ingresos más altos e ingresos más bajos era más del doble.

- En el trabajo Quality of life in Europe (European Foundation for the Improvement of Living and Working Conditions, 2014) el porcentaje de personas que contestaron que nunca se han sentido solas varía entre el $35 \%-45 \%$ de países como Italia, Polonia o Grecia, el $48 \%-56 \%$ de países como España, Bélgica, Portugal, Francia, y países como Alemania, Finlandia, Austria, Holanda o Dinamarca, cuyo porcentaje de personas que nunca se han sentido solas varía entre un $59 \%-75 \%$. En general, parece existir un patrón que más adelante se comprueba en otras investigaciones: la soledad es mayor en el sur de Europa que en el norte; mayor en el este que en el oeste de Europa, es decir, sociedades más familiaristas (mayor soledad) frente a sociedades más individualistas (menor soledad).

- La soledad parece ser mayor al final de la adolescencia y en la vejez que en otros momentos del ciclo vital (Luhmann y Hawkley, 2016) y ha sido relacionada con indicadores de integración social como las relaciones de pareja, el apoyo social percibido y la aceptación (Adam et al., 2014). En general, la investigación asume que las personas solteras se sienten más solas que las casadas (Luhmann y Hawkley, 2016) y no tener hijos parece no tener un efecto directo sobre la soledad en mayores, aunque vivir solo se relaciona con una menor red social, que es un indicador de soledad tanto en los hombres como en las mujeres. En cuanto al género, la evidencia empírica es contradictoria: hay investigaciones que no encuentran diferencias (Zebhauser et al., 2011), mientras que otras (Tesch-Römer et al., 2013) hallaron mayores índices de soledad en hombres que en mujeres. Y en otras investigaciones (Zebhauser et al., 2014) se encontró que el nivel medio de soledad solo difería en los mayores de 85 años, donde la soledad fue mayor en mujeres que en hombres. En nuestro caso particular, en un estudio del Programa de Mayores de la Fundación Bancaria la Caixa (Yanguas et al., en prensa) con una muestra de 550 personas mayores, encontramos en una muestra de personas que acuden a centros de mayores, tasas de soledad mayores en hombres que en mujeres, siendo estadísticamente significativas dichas diferencias.

- Como antes se ha insinuado, las tasas de soledad suelen ser más elevadas en los países del este y sur de Europa que en el norte (del Barrio et al., 2010; Dykstra, 2009; Fokkema, de Jong Gierveld y Dykstra, 2012; Rico-Uribe et al., 2016; Sánchez- Rodríguez et al., 2012; Yang y Victor, 2011; de Jong Gierveld y Tesch-Römer, 2012; Hansen y Slagsvold, 2015). Por ejemplo, según una investigación transcultural en doce países europeos (Sundström et al., 2009): Suiza, Dinamarca y Suecia presentaron las tasas más bajas de soledad; las más altas se 
encontraron en Francia, Israel, Italia y Grecia; la prevalencia de soledad casi todo el tiempo varió de un $1 \%$ en Suiza a un $10 \%$ en Grecia, siendo un $7 \%$ en España. En otras palabras: las sociedades más familiaristas tienen mayores tasas de soledad, que las sociedades más individualistas.

- En España y Latinoamérica los estudios escasean, pero Andrés Losada y colaboradores de la Universidad Rey Juan Carlos de Madrid (Losada et al., 2012) hallaron que el 23,1 \% de su muestra de personas mayores españolas se sentían solas, siendo que el $17,1 \%$ se sentían solas a veces y el $6 \%$ a menudo; y VelardeMayol et al. (2015) hallaron que la mayoría de los que vivían solos -el 63,2 \%-presentaron niveles de soledad entre moderados y graves. Al comparar la muestra de personas que vivían solas con la de las que vivían acompañadas, casi dos tercios de las primeras experimentaban mayor grado de soledad que aquellas que no vivían solas. SánchezRodríguez (2009) halló tasas de soledad de un 58,7 \% (presentaban algún grado de soledad), el $42 \%$ de la muestra informó de niveles bajos de soledad, mientras que el 16,8 \% sufría soledad moderada o grave. En otro estudio que se publicará próximamente realizado por este Programa de Mayores con una muestra de 1.688 mayores de 20 años en ocho ciudades españolas (Yanguas et al., en prensa), se encontraron porcentajes de "riesgo" de aislamiento social cercanos al 39 $\%$ de la población y tasas de soledad cercanas al $24 \%$ que varían según diversas variables sociodemográficas.

- Asimismo, se han encontrado diferencias en el nivel socioeconómico, el estado de salud y las redes sociales de las personas que viven en distintos países que podrían facilitar o dificultar sus oportunidades de participar en actividades sociales y de contar con relaciones sociales satisfactorias (Hansen y Slagsvold, 2015). Y se ha constatado la influencia de los valores culturales en los diferentes países en la personalidad de sus habitantes, lo que afectaría a las expectativas que tienen sobre sus relaciones sociales y consecuentemente contribuiría a una mayor o menor prevalencia de soledad (Yang y Victor, 2011).

Creemos que se puede afirmar que la soledad es un problema prevalente y serio (como intentaremos demostrar en el punto siguiente), que irá en aumento en las próximas décadas con un coste personal, social y sanitario de primer orden, además de muy injusto.

\section{Consecuencias de la soledad}

La evidencia empírica que describe la influencia de la soledad en la salud tanto física como psicológica es abrumadora (Constança, 2014; Yanguas, 2018;
Yanguas, Pinazo y Tarazona, 2018) y en ella se ha centrado una gran parte de la labor investigadora: conocer las consecuencias de la soledad en distintas áreas de funcionamiento de un individuo.

Así la soledad tiene influencia en ambas dimensiones:

- En la salud física:

- Empeora el funcionamiento vascular (Cacioppo, Hawkley, Crawford et al., 2002; O’Luanaigh y Lawlor, 2008).

- Aumenta la presión sistólica (Hawkley, Masi et al., 2010).

- Acrecienta la posibilidad de sufrir accidentes vasculares recurrentes (Cacioppo, Capitanio y Cacioppo, 2014).

- Disminuye la expresión de genes ligados a la respuesta antinflamatoria y aumentan la sobre expresión de genes asociados a la proinflamación (Cole et al., 2007).

- Amplifica la actividad del eje hipotalámicopituitario-adrenal HPA (Adam et al., 2006; Steptoe et al., 2004), esencial en los procesos de estrés.

- Eleva las alteraciones en el sistema inmune (Pressman et al., 2005) y empeora la nutrición (Ramic et al., 2011).

- Acentúa la obesidad (Lauder et al., 2006).

- Amplifica el declive motor (Buchman et al., 2010).

- Potencia la reducción de la actividad física y la capacidad funcional (Shiovitz-Ezra y Ayalon, 2010).

- Influencia la relación entre los genes y el ambiente (Goossens et al., 2015; Gao et al., 2017).

- En la salud psicológica:

- Predice síntomas depresivos (Cacioppo et al., 2006; Holwerda et al., 2012).

- Aumenta los problemas de sueño (Cacioppo, Hawkley, Berntson et al., 2002).

- Empeora el funcionamiento cognitivo y aumenta el riesgo de padecer alzhéimer (Wilson et al., 2007), además de empeorar las puntuaciones en tests neuropsicológicos en distintas funciones cognitivas, entre ellas: memoria inmediata, memoria visual, memoria episódica, memoria semántica, velocidad de procesamiento y función ejecutiva (Zhong et al., 2017; Boss, Kang y Branson, 2015).

- Acrecienta los problemas de salud mental (Tylova et al., 2013).

- Incrementa la tasa de institucionalización (Russell et al., 1997; Logman et al., 2013).

- Eleva la mortalidad (Steptoe et al., 2013; Luo et al., 2012).

- Algunos autores creen que la soledad funciona como un riesgo de salud, similar a los establecidos como factores de riesgo "clásicos", como la obesidad, el colesterol, etc. (Holt-Lunstad et al., 2010), además de 
generar sufrimiento y disminuir la calidad de vida.

Ante tanta consecuencia negativa, también conviene matizar. Algunas autoras (Birditt et al., 2018) han comprobado que la soledad puede ser menos lesiva que mantener relaciones sociales conflictivas; es decir, las personas con redes sociales más conflictivas se beneficiaron de la soledad al mostrar un menor afecto negativo (cuando están solos) en comparación con los individuos con redes menos conflictivas. Así, mantener la soledad puede ser una solución eficaz para personas con redes sociales negativas al poder utilizar la soledad (consciente o inconscientemente) como mecanismo de afrontamiento para evitar relaciones sociales potencialmente dañinas. En otras palabras, el proverbio, "mejor solo que mal acompañado" parece de gozar de refrendo experimental.

Recapitulando, el sufrimiento y el dolor que la soledad genera correlaciona ampliamente tanto con la salud física como con la salud psicológica y, sin ninguna duda, se puede afirmar que la soledad, además de algo muy injusto cuando una persona llega a la vejez (obviamente también en cualquier edad), es un problema de salud serio, prevalente (Cacioppo y Patrick, 2008; Hawkley et al., 2010), con innumerables consecuencias negativas y con un coste económico - todavía no cuantificado- que se presume muy elevado.

\section{Intervenciones en soledad}

\subsection{Análisis general}

Las publicaciones sobre intervenciones en soledad (Yanguas, 2018) y aislamiento social en mayores que muestren datos de evaluación y medición del efecto del programa en los participantes son escasas, con muestras pequeñas y metodología habitualmente incompleta (habitualmente sin grupos de control, etc.). Se puede destacar que:

- Según diversos artículos, las intervenciones grupales, con actividades de apoyo y educativas, dirigidas a grupos específicos y que se apoyan en los recursos comunitarios existentes, que incluyen además un entrenamiento y apoyo específico a los facilitadores (Findlay, 2003; Cattan et al., 2005; Dickens et al., 2011) generalmente voluntarios de estas acciones, parecen ser las intervenciones más eficaces.

- Cuatro estrategias de intervención son las que cuentan con más refrendo científico (Masi et al., 2011): las que aumentan las habilidades sociales; las dirigidas a fortalecer el apoyo social; las que aumentan las oportunidades de interacción social; y las dirigidas al entrenamiento sociocognitivo.

- Las publicaciones existentes constatan el uso de muy diversos diseños metodológicos: desde diseños cuasi experimentales más "ligeros" con o sin grupo control, evaluación pre y post intervención, para medir la eficacia diferencial de tres tipos de programas de intervención (Bartlett et al., 2013), hasta ensayos aleatorizados controlados, con grupo experimental y grupo de control, con medidas pre y post intervención (a los tres y seis meses) de mucha mayor consistencia metodológica (Pitkala et al., 2009). Además, es cada vez más común, aunque minoritario, el uso de metodología cualitativa para la evaluación de programas de intervención contra la soledad no deseada y el aislamiento (Hemingway y Jack, 2013; Swindells, 2013); las llamadas telefónicas en Call in Time, de Help the Aged (Banks y Banks, 2002); y distintos proyectos que han evaluado sus intervenciones con metodologías mixtas (cuantitativa y cualitativa) en el mismo proceso evaluativo (Yanguas et al., 2018).

- En la evaluación de intervenciones en soledad se han utilizado distintos instrumentos de medida, lo que dificulta hacer comparaciones de eficacia entre intervenciones. No es lo mismo utilizar la UCLA en sus diferentes versiones -UCLA, UCLA-R, UCLA-3- (Banks y Banks, 2002), que la escala De Jong Gierveld (Fokkema y Knipscheer, 2007; CollPlanas et al., 2017), el convoy de relaciones (Kahn y Antonucci, 1980), la escala de redes sociales de Lubben (1988), o algunas escalas de estado de ánimo como el Panas para medir bienestar subjetivo, debido esencialmente a que las pruebas que miden soledad o red social, no están midiendo el mismo constructo ni constitutivo ni operativo, por lo cual los resultados son difícilmente comparables (Yanguas, 2018).

- En lo relativo al formato de la intervención y su eficacia, existe un buen número de revisiones sistemáticas (Findlay, 2003; Cattan et al., 2005; Hagan et al., 2014). Cattan et al. (2005) identificaron y categorizaron treinta estudios, siendo diecisiete de ellos intervenciones de tipo grupal, diez intervenciones individuales, tres provisiones de servicios y una sobre desarrollo comunitario. El $90 \%$ de las intervenciones eficaces fueron intervenciones grupales con grupo de apoyo o educativo, mientras que seis de las ocho intervenciones ineficaces proporcionaron apoyo individualizado (basado en consejo, información o asesoramiento en necesidades de salud). En general, se acepta que las intervenciones grupales educativas y sociales dirigidas a grupos específicos pueden aliviar el aislamiento social y la soledad en las personas mayores, mientras que la efectividad de las intervenciones centradas en las visitas a domicilio y befriending (fomento de la amistad, compañía, etc.) no ha sido demostrada (lo cual no significa que carezcan de importancia). Por otro lado, Stojanovic et al. (2016) realizaron una revisión y puesta al día de intervenciones en soledad y apoyo social publicadas en castellano e italiano: de las quince analizadas, ocho mostraron una reducción de la soledad y seis un aumento 
importante en apoyo social y reducción del aislamiento social. De entre ellas, los programas comunitarios mostraron un importante papel en el aumento de la inclusión social, mientras que las intervenciones educativas centradas en la implicación social y el apoyo fueron eficaces para reducir la soledad.

- Las intervenciones basadas en tecnologías y los programas de actividad física aparecen como una alternativa prometedora, aunque diversos autores han dado la voz de alarma sobre la necesidad de hacer "buenas" intervenciones y no solo intervenciones "tecnológicamente innovadoras" (Poscia et al., 2016; Yanguas, Pinazo y Tarazona, 2018), que es el gran peligro en este momento. La mayor parte de estas intervenciones con base tecnológica se centran en el fortalecimiento de los vínculos sociales y de amistad, su facilidad y bajo coste, además de sus grandes posibilidades para aumentar las redes sociales y reducir hipotéticamente "la soledad", así como la apuesta en Europa por los usos sociales y asistenciales les han dado alas. La evidencia empírica existente, sin embargo, parece cuestionar algunas de las bondades de este tipo de sistemas: Pitkala et al. (2009) encontraron resultados alentadores sobre la eficacia, pero más recientemente Chipps, Jarvis y Ramlall (2017), tras realizar una revisión sistemática de "e-intervenciones" identificaron que algunos programas obtenían una eficacia moderada y que los estudios que los avalaban mostraban falta de rigor, concluyendo que la evidencia de este tipo de intervenciones es inconsistente y débil, por el momento.

- En la mayoría de los textos analizados, el contexto de las intervenciones es fundamentalmente comunitario, aunque sí es cierto que hay algunas intervenciones en contextos específicos, por ejemplo, en residencias de personas mayores. De estas, algunas utilizan la terapia asistida por animales, como el programa de Banks y Banks (2002) o el de Vrbanac et al. (2013) con sesiones semanales con perros. Otras utilizan ejercicio físico (Tse et al., 2014) o programas de jardinería en interiores para facilitar el aumento de la socialización y la satisfacción con la vida, reducir la soledad y promover actividades de la vida diaria de las personas mayores que viven en residencias (Brown et al., 2004). En cualquier caso, y como destacan algunos autores, las intervenciones en soledad parecen estar ausentes de los centros residenciales, aunque la prevalencia de esta sea abrumadora (Jansson et al., 2017; Drageset et al., 2011; Slettebø, 2008), llegando en algunos casos a casi el $60 \%$ de las personas que residen en ellas.

Como destacan diversos autores (Findlay, 2003; Masi et al., 2011; Cohen-Masfield y Perach, 2015; Ong et al., 2016) tenemos más programas de intervención que evidencias de sus bondades, debido a falta de rigor metodológico y deficiencias evaluativas; aproximaciones muy variadas (cognitivas, fomento de relaciones, compañía, incrementar oportunidades de relación, tecnologías, etc.) que hacen muy complicada su comparación; y una falta de multidimensionalidad en la manera de entender las intervenciones en soledad, que por ejemplo no recoge el necesario empoderamiento de las personas para que aprendan a gestionar y vivir su propia soledad.

\subsection{La propuesta del programa "Siempre Acompañados"}

El Programa “Siempre Acompañados” nació en noviembre del 2013 dentro del Programa de Mayores de la Fundación Bancaria la Caixa en colaboración con la Cruz Roja de Cataluña, para dar respuesta a las situaciones de soledad no deseada desde las siguientes premisas fundamentales:

- Comprender e intervenir en soledad desde su complejidad: siendo la soledad un sentimiento, se busca una intervención que aborde su comprensión; siendo la soledad una discrepancia, se procura implementar una intervención que, combinada con la actuación a nivel emocional "traccione" del cambio cognitivo; si la soledad, además de personal, tiene relación con la falta de vínculos (es “comunitaria”), se incide en la generación de comunidades comprometidas con ella, a la vez que se sigue trabajando en la intervención individual; si la soledad se explica por el mantenimiento de normas, valores o estereotipos negativos, se busca conjuntamente con la ciudadanía transformarlos; si la soledad es provocada por múltiples motivos y la persona le hace frente a través de innumerables formas diferentes, se intentan mejorar los modos de afrontamiento; si la soledad se presenta combinada con otras situaciones que se dan en la vejez (fragilidad, pérdidas, vulnerabilidad, etc.), se explora la posibilidad de una intervención combinada.

- Dar respuesta a la complejidad del fenómeno reclama una intervención a múltiples niveles y esto implica:

- Trabajar con las personas en situación de soledad. El funcionamiento social y la soledad, dependen (no exclusivamente) de lo que "hace" la persona y, como sucede en otras esferas de la vida (salud física, por ejemplo), existe una responsabilidad individual de cuidar las relaciones sociales y gestionarse cada uno (en su caso) su propia soledad, que no es posible eludir. El programa pretende ofrecer oportunidades para que las personas se empoderen, para que sean capaces de gestionar sus relaciones y su propia soledad, desde la confianza en ellas y en sus capacidades, trabajando conjuntamente con ellas. La intervención individual surge desde el conocimiento de la persona y su proyecto 
personal, y pasa por su empoderamiento e implicación, coordinando e integrando acciones.

- Trabajar con las comunidades. Generar arquitecturas comunitarias y, en la medida de lo posible, redes de apoyo, bienestar y cuidado entre los ciudadanos, impulsando procesos de participación social y capacitando a las personas y a las entidades para afrontar retos que les son comunes. La soledad y las relaciones sociales están (como se ha abordado anteriormente) vinculadas al sentimiento de pertenencia, a la posibilidad de mutua influencia, a la satisfacción de necesidades propias y comunes, y a la conexión emocional compartida.

- Sensibilizar a la ciudadanía, generando interdependencia y cuidado mutuo, tanto en cuestiones relativas a la soledad en particular, como hacia el envejecimiento y las situaciones de vulnerabilidad en general.

- Buscar soluciones desde los territorios, desde su idiosincrasia propia, desde las historias personales. Las intervenciones del programa Siempre Acompañados no son un "catálogo" de recursos fijo ("tenga usted lo que tenga, sienta usted lo que sienta, sean sus recursos personales distintos de los de otra persona, y su comunidad diferente", etc. le aplicamos lo que tenemos en catálogo), sino un proyecto que busca intermediar, "negociar”, implementar soluciones innovadoras en cada caso, consensuadas con los participantes (persona, comunidad, ciudadanía y profesionales), un proyecto comprometido con una intervención centrada en las personas (persona en situación e soledad, voluntarios, asociaciones, etc.).

- La soledad reclama un abordaje común entre Administraciones Públicas, entidades, asociaciones y personas que, desde el territorio y respetando sus particularidades, interaccionan y trabajan en común (con metodologías elaboradas el efecto) en la búsqueda consensuada de un objetivo. Por ello, el impulso del voluntariado, la sensibilización vecinal y la implicación de los profesionales en estructuras comunitarias de trabajo son otra cuestión fundamental de este programa.

- Para poder acometer un abordaje de este tipo, se han creado, desarrollado y validado diversos elementos metodológicos:

- Para el conocimiento y evaluación de personas y comunidades.

- Para la realización de planes de intervención conjuntos entre personas, profesionales y voluntarios.

- Para crear espacios de trabajo comunitario (grupos de acción social) en los que se articulan acciones de respuesta desde los recursos de la propia comunidad a las necesidades de las personas en situación de soledad, así como a la organización de la propia comunidad.

- El programa, que actualmente está en marcha en ocho municipios del Estado comenzó en Terrassa, Tortosa y Girona (desde el 2013 donde se realizó el piloto) y desde finales del 2017 se ha hecho extensivo a Tárrega, Santa Coloma de Gramenet, Logroño, Palma y Jerez de la Frontera, con una intención de extensión a otros territorios. La prueba piloto fue evaluada externamente por el Instituto de Gobierno y Políticas Publicas-IGOP de la Universidad Autónoma de Barcelona, cuyos principales resultados, ya aplicados al resto de territorios, son los siguientes:

- Importancia de crear y articular en la intervención en soledad (a nivel comunitario) lo que hemos venido a llamar Grupos de Acción Social como grupos de trabajo en los que participan una diversidad de agentes -tanto profesionales como de la ciudadanía-, que facilitan la creación de espacios inéditos de conocimiento, reflexión y trabajo conjunto que permiten una mirada más completa de las personas mayores, de los mayores en situación de soledad, así como actuaciones más diversas y complejas. En estos grupos tenemos: ayuntamientos (departamentos de servicios sociales, seguridad ciudadana, etc.), gobiernos autonómicos, hospitales y centros de salud, entidades sociales (Cáritas, Cruz Roja, Amics de la Gent Gran, etc.), centros de personas mayores, centros cívicos, asociaciones de personas mayores, asociaciones de vecinos y comerciantes, colegios profesionales (farmacéuticos, etc.), parroquias, agente de la propiedad inmobiliaria, y un largo etcétera.

- Aumentar y mejorar (en términos de calidad) la presencia e implicación de la ciudadanía en los espacios de trabajo sigue siendo uno de los principales retos de un programa de este tipo, al facilitar la relación positiva entre recursos técnicos y no técnicos, aumentando la capacidad de aportación tanto en la planificación de acciones, como en la sensibilización.

- Datos extraídos de este estudio piloto (así como de otros antes comentados en el tercer punto de este artículo) confirman la existencia de estereotipos extendidos en la población tanto a la vejez en general, como a las personas mayores en situación de soledad, que bloquean y crean resistencias en la participación de las personas, que deben ser superados

- Con frecuencia, la soledad se alimenta de otras situaciones de fragilidad y vulnerabilidad. El programa "Siempre Acompañados" ayuda a visibilizar otras problemáticas asociadas a la soledad de las personas mayores y fomenta el trabajo conjunto sobre ellas (agentes diversos trabajando en común), adaptando los recursos 
y las actividades a sus necesidades, así como empoderando personas en contextos de confianza para ellas.

- Importancia de un buen diagnóstico de las personas mayores en situación de soledad, que permiten a las personas que sufren soledad hacerse conscientes de su situación, reflexionar y profundizar, entendiendo este paso como fundamental antes de iniciar cualquier actuación que implique el empoderamiento de las mismas.

- Necesidad de profundizar en el trabajo con las familias en el caso de que las haya, como agentes especialmente relevantes. El trabajo con las familias de personas en situación de soledad es especialmente sensible y delicado, y necesita un abordaje diferencial.

- Se ha rediseñado la intervención individual (que se adapta a cada situación) que está enfocada en tres fases diferentes:

I. Una primera de valoración y puesta en marcha, que desde la confianza entre el profesional y la persona se inicia con una evaluación o diagnóstico, concluyendo con la elaboración conjunta (profesionales, persona, voluntarios) y puesta en marcha del plan de intervención.

II. Una segunda fase de seguimiento tanto a la persona, como a la red comunitaria que la apoya, que incluye reevaluaciones de la situación y reelaboraciones de los planes de intervención conjuntos consensuados.

III. Una tercera fase donde se pretende (aunque no siempre es viable) que el individuo se integre en la comunidad y deje el programa.
- La experiencia de estos últimos años ha subrayado la necesidad de ahondar en la formación de profesionales, voluntarios y ciudadanía - a distintos niveles, aunque complementariamente-sobre las relaciones sociales y la soledad.

- Hasta la fecha se ha atendido a 374 personas en situación de soledad y más de 100 entidades se han integrado de manera activa al programa.

\section{Conclusiones}

En este artículo hemos pretendido delimitar lo que es la soledad en las personas mayores, conocer a quiénes y a cuántos afecta, indagar en sus causas y en sus consecuencias, así como analizar la eficacia de las intervenciones existentes, presentando brevemente la propuesta del programa "Siempre Acompañados".

Cuando evaluamos personas en situación de soledad les preguntamos por "si se sienten abandonadas", "si se sienten infelices", "si creen que nadie les comprende", "si se sienten completamente solas", "si ansían la compañía de otras personas"... Todas estas preguntas evocan, siquiera por su ausencia, solidaridad, interdependencia, empatía, comprensión, paciencia, agradecimiento, etc., valores esenciales de toda sociedad sana. La soledad no es solo uno de los mayores retos a los que se enfrenta el mundo del envejecimiento en las próximas décadas (que es lo mismo que decir la sociedad en general), sino una oportunidad de innovación social, de progreso tanto de las personas como de las sociedades; y estar solo (si no es deseado) retrata concienzudamente la perversidad de un mundo que reclama cambios. 


\section{Bibliografía referenciada}

AARTSEN, M. y JYLHA, M. (2011): “Onset of loneliness in older adults: Results of a 28 year prospective study", European Journal of Ageing, 8(1), 31-38 [〈http://doi.org/10.1007/s10433-011-0175-7)]

ADAM, E.K. et al. (2011): “Adverse adolescent relationship histories and young adult health: cumulative effects of loneliness, low parental support, relationship instability, intimate partner violence, and loss", The Journal of adolescent health: official publication of the Society for Adolescent Medicine, 49(3), 278-86.

ADAM E.K. et al. (2006): "Day-to-day dynamics of experience--cortisol associations in a population-based sample of older adults", Proceedings of the National Academy of Sciences, 103, 17058-63 [rhttp://doi. org/10.1073/pnas.0605053103)].

AJROUCH, K.J.; BLANDON, A.Y. y ANTONUCCI, T.C. (2005): "Social networks among men and women", Journals of Gerontology Series B: Psychological Sciences and Social Sciences, 60, 311-317.

BANKS, M.R. y BANKS, W.A. (2002): The effects of animalassisted therapy on loneliness in an elderly population in long-term care facilities, The journals of gerontology. Series A, Biological sciences and medical sciences, 57(7), 428-432.

BARTLETT, H. et al. (2013): "Preventing social isolation in later life: Findings and insights from a pilot Queensland intervention study", Ageing \& Society, 33, 1.167-1.189.

BIRDITT, K.S. et al. (2018): "Better Off Alone: Daily Solitude Is Associated With Lower Negative Affect in More Conflictual Social Networks", The Gerontologist, gnyo6o, [<https://doi. org/10.1093/geront/gnyo60)]

BOSS, L.; KANG, D.H.; y Branson, S. (2015): “Loneliness and cognitive function in the older adult: a systematic review", International

Psychogeriatrics, 27(4):541-553.

BROWN, V.M. et al. (2004): “Indoor gardening and older adults: Effects on socialization, activities of daily living and loneliness", Journal of Gerontological Nursing, 30:34-42.

BUCHMAN, A.S. et al. (2010): "Loneliness and the rate of motor decline in old age: the Rush Memory and Aging Project, a community-based cohort study", BMC Geriatrics, 10, 77.

CABLE, N. et al. (2013): "Friends are equally important to men and women, but family matters more for men's wellbeing", Journal of Epidemiology \& Community Health, 67, 166-171.

CACIOPPO, J.T. et al. (2002): “Do lonely days invade the nights? Potential social modulation of sleep efficiency", Psychological Science, 13 (4), 384387.

CACIOPPO, S.; CAPITANIO, J.P. y CACIOPPO, J.T. (2014): "Toward a neurology of loneliness", Psychological Bulletin [<https://doi. org/10.1037/a0037618>].

CACIOPPO, J.T. y PATRICK, W. (2008): “Lonely in a social world”, en: CACIOPPO, J. T. y PATRICK, W. (Eds.): Loneliness: human nature and the need for social connection, 3-19.

CACIOPPO, J.T. et al. (2006): “Loneliness as a specific risk factor for depressive symptoms: cross-sectional and longitudinal analyses", Psychology and Aging, 21, 1, 140-151.

CARSTENSEN, L.L. (1992): "Social and emotional patterns in adulthood”, Psychology and Aging, 7, 331-338.

CATTAN, M. et al. (2005): "Preventing social isolation and loneliness among older people: a systematic 
review of health promotion interventions", Ageing \& Society, 25 (1):41-67.

CHIPPS, J.; JARVIS, M.A. y RAMLALL, S. (2017): “The effectiveness of e-Interventions on reducing social isolation in older persons: A systematic review of systematic reviews"; I Telemed Telecare, 23(10): 817-827 [<https://doi. org/10.1177/1357633X17733773'].

COHEN-MANSFIELD, J. y PERACH, R. (2015): “Interventions for alleviating loneliness among older persons: a critical review", Am J Health Promot, 29: 109125.

COLE, S.W. et al. (2007): Social regulation of gene expression in human leukocytes, Genome Biology, 8(9), R189.181-R189.113 ['https://doi.org/10.1186/ gb-2007-8-9-r189<].

COLL-PLANAS, L. et al. (2017): "Promoting social capital to alleviate loneliness and improve health among older people in Spain", Health and Social Care in the Community, 25 (1), 145-157.

CONSTANÇA, P. (2014): "Loneliness and health in later life", en PACHANA N.A. y LAIDLAW, K. (eds.): The Oxford handbook of Clinical Geropsychology, Oxford University Press.

CORNWELL, B.; LAUMANN, E.O. y SCHUMM, L.P. (2008): The social connectedness of older adults, American Sociological Review, 73, 185-203.

CUMMING, E. y HENRY, W. (1961): Growing old, New York, Basic Books.

DAHLBERG, L.; ANDERSSON, L. y LENNARTSSON, C. (2016): "Long-term predictors of loneliness in old age: results of a 20-year national study", Aging \& Mental Health. [khttp://dx.doi.org/10.1080/136 07863.2016.1247425>].

DALTON, J.H.; ELIAS, M.J. y WANDERSMAN, A. (2001): Community Psychology. Linking Individuals and Communities, California, Wadsworth.

DE JONG GIERVELD, J. (1987): "Developing and testing a model of loneliness", J of Pers and Social Psych, 53, 119-128.

DE JONG GIERVELD, J.; VAN DER PAS, S. y KEATING, N. (2015): "Loneliness of older immigrant groups in Canada: Effects of ethnic-cultural background", Journal of Cross-Cultural Gerontology, 30 , 251-268 [rhttps://doi.org/10.1007/s10823-0159265-x>].

DE JONG GIERVELD, J. y TESCH-RÖMER, C. (2012): "Loneliness in old age in Eastern and Western European societies: Theoretical perspectives", European Journal of Ageing, 9, 285-295, [rhttps://doi.org/10.1007/s10433-012-02482)].

DEL BARRIO et al. (2010): "La soledad de las personas mayores en España y Suecia: Contexto y cultura”, Revista Española de Geriatría y Gerontología, 45, 4, 189-195.

DICKENS, A.P. et al. (2011): "Targeting social isolation in older people: A systematic review", BMC Public Health, 11, 1-22.

DONIO BELLEGARDE, M. (2017): La soledad de las mujeres mayores que viven solas, tesis doctoral no publicada, Universidad de Valencia.
DRAGESET, J. et al. (2011): "Loneliness and social support among nursing home residents without cognitive impairment: A questionnaire survey", International Journal of Nursing Studies, 48, 5, 611-619.

Dykstra, P. A. (2009): Older adult loneliness: Myths and realities. European Journal of Ageing, 6, 91-100 [/https://doi.org/10.1007/s10433-009-0110-3/].

EUROPEAN FOUNDATION FOR THE IMPROVEMENT OF LIVING AND WORKING CONDITIONS (2014): Third European quality of life survey - Quality of life in Europe: Trends 2003-2012, Luxemburgo, Publications Office of the European Union.

FINDLAY, R.A. (2003): “Interventions to reduce social isolation amongst older people: where is the evidence?", Ageing Society, 23: 647-658.

FOKKEMA, T.; DE JONG GIERVELD, J. y Dykstra, P.A. (2012): "Cross-national differences in older adult loneliness", The Journal of Psychology: Interdisciplinary and Applied, 146, 1-2, 201-28.

GAO et al. (2017): “Genome-Wide Association Study of Loneliness Demonstrates a Role for Common Variation", Neuropsychopharmacology, 42, 811-821.

GOOSSENS, L. et al. (2015): "The genetics of loneliness: Linking evolutionary theory to genomewide genetics, epigenetics, and social science", Perspectives on Psychological Science, 10, 213-226.

HAGAN, R. et al. (2014): "Reducing loneliness amongst older people: a systematic search and narrative review", Aging \& Mental Health, 18(6), 683-693. [khttps://doi.org/ $10.1080 / 13607863.2013 .875122\rangle]$.

HANSEN, T. y SLAGSVOLD, B. (2015): "Late-life loneliness in 11 European counties: Results from the Generations and Gender Survey", Social Indicators Research, 1-20.

HAUGE, S. y KIRKEVOLD, M. (2010): “Older Norwegians' understanding of loneliness", International Journal of Qualitative Studies on Health and Well-being, 5, 4654.

HAWKLEY, L.C. y CAPITANIO, J.P. (2015): “Perceived social isolation, evolutionary fitness and health outcomes: a lifespan approach", Philosophical Transactions of the Royal Society B: Biological Sciences, 370 (1669): 20140114.

HAWKLEY, L.C.; MASI, C.M. y CACIOPPO, J.T. (2010): "Loneliness predicts increased blood pressure: 5-year cross-lagged analyses in middle-aged and older adults", Psychology and Aging, 25(1): 132-141.

HEMINGWAY, A.; JACK E. (2013): "Reducing social isolation and promoting well-being in older people", Quality in Ageing and Older Adults, 14 (1), 25-35. [rhttps://doi.org/10.1108/14717791311 311085'].

HOJAT, M. (1989): “A Psychodynamic View of Loneliness and Mother-Child Relationship: A Review of Theoretical Perspectives and Empirical Findings", en HOJAT, M., y CRANDALL, R. (eds.): Loneliness: Theory, research, and Applications 89-104, Sage. 
HOLT-LUNSTAD, J.; SMITH, T.B. y LAYTON, J.B. (2010): “Social Relationships and Mortality Risk: A Metaanalytic Review", PLOS Medicine, 7(7) [rhttps:// doi.org/10.1371/ journal.pmed.1000316〉].

HOLWERDA, T.J. et al. (2012): « Feelings of loneliness, but not social isolation, predict dementia onset: results from the Amsterdam Study of the Elderly (AMSTEL)", Journal of Neurology, Neurosurgery, and Psychiatry, 0, 1-8 [rhttps:// doi.org/10.1136/jnnp-2012-302755'].

Instituto Nacional de Estadística (2015): Encuesta continua de hogares, Instituto Nacional de Estadística, Madrid.

- (2017): Encuesta continua de hogares, Instituto Nacional de Estadística, Madrid.

JANSSON, A.H. et al. (2017): "Loneliness in nursing homes and assisted living facilities: prevalence, associated and prognosis", The Journal of Nursing Home Research, 3, 43-49.

KAHN, R.L. y ANTONUCCI, T. C. (1980): "Convoys over the life course: Attachment, roles, and social support”, en Baltes, P.B. y BRIM, O. (eds.): Lifespan development and behavior, 3, 254-283, Academic Press.

LAUDER, W. et al. (2006): A comparison of health behaviours in lonely and non-lonely populations, Psychology, Health \& Medicine, 11, 233-245 [rhttps://doi.org/10.1080/135485005002666 07)].

LONGMAN, J. et al. (2013): "The role of social isolation in frequent and/or avoidable hospitalisation: rural community-based service providers' perspectives", Australian Health Review, 37 (2): 223-231.

LOSADA, A. et al. (2012): "Loneliness and mental health in a representative sample of community-dwelling Spanish older adults", Journal of Psychology: Interdisciplinary and Applied, 146, 3, 277-292.

LUBBEN, J. et al. (2016): "Performance of an abbreviated version of the Lubben Social Network Scale among three European community-dwelling older adult populations", The Gerontologist, vol. 46, p. 11, 2006.

LUBBEN, J. (1988): “Assessing social networks among elderly populations”, Family \& Community Health: The Journal of Health Promotion \& Maintenance, 11, 42-52.

LUHMANN, M y HAWKLEY, L.C. (2016): “Age Differences in Loneliness From Late Adolescence to Oldest Old Age", Developmental Psychology, 62 (6), 943-59.

LUO, Y. et al. (2012): "Loneliness, health, and mortality in old age: A national longitudinal study", Social Science \& Medicine [rhttps://doi.org/74:907 914.10.1016/j.socscimed.2011.11.028>].

MAIS, C.M. et al. (2011): "A meta-analysis of interventions to reduce loneliness", Pers Soc Psychol Rev, 15(3), 219-266 [rhttps://doi. org/10.1177/1088868310377394.

MUSHTAQ, R. et al. (2014): "Relationship between loneliness, psychiatric disorders and physical health? A review on the psychological aspects of loneliness", Journal of clinical and diagnostic research, 8, 9 .
O'LUANAIGH, C. y LAWLOR, B.A. (2008): "Loneliness and the health of older people", International Journal of Geriatric Psychiatry, 23 (12), 1213-1221.

ONG, A.D.; UCHINO, B.N. y WETHINGTON, E. (2016): "Loneliness and Health in Older Adults: A Mini-Review and Synthesis", Gerontology, 62, 443-449.

PAHL, R. y PEVALIN, D.J. (2005): “Between family and friends: A longitudinal study of friendship choice", British Journal of Sociology, 56, 433450.

PEPLAU, L.A. y PERLMAN, D. (1982): Loneliness: $a$ sourcebook of current theory, research and therapy, John Wiley and Sons.

PIKHARTOVAA, J.; BOWLING, A.; Victor, C. (2016): "Is loneliness in later life a self-fulfilling prophecy?", Aging \& Mental Health, 20, 5, 543549 [<http://dx.doi.org/10.1080/13607863.20 15.1023767>].

PINAZO, S. y DONIO BELEGARDE, M. (2108): La soledad en las personas mayores, Fundación Pilares, Madrid.

PITKALA, K.H. et al. (2009): "Effects of psychosocial group rehailitation on health, use of health care services, and mortality o folder personas suffering from loneliness: a randomized, controlled trial", The journals of gerontology. Series A, Biological sciences and medical sciences, 64, 792-800.

POSCIA, A. et al. (2016): "Interventions targeting loneliness and social isolation among the older people: An update systematic review", Experimental Gerontology, 102:133-144.

PRESSMAN, S.D. et al. (2005): “Loneliness, social network size, and immune response to influenza vaccination in college freshmen", Health Psychology, 24 (3), 297-306.

RAMIC, E. et al. (2011): "The effect of loneliness on Malnutrition in elderly population", Medical Archives, 65 (2), 92-95.

RICO-URIBE, L.A. et al. (2016): “Loneliness, social networks, and health: A cross-sectional study in three countries", PLOS ONE, 11, 1.

RUBENSTEIN, C.M. y SHAVER, P. (1980): "Loneliness in two northeastern cities", en HARTOG J., AUDY J.R. y COHEN Y.A. (eds.), The anatomy of loneliness (319-337). New York, International Universities Press.

RUBENSTEIN, C. y SHAVER, P. (1982): The experience of loneliness, en PEPLAU, L.P. y PERLMAN, D. (eds.): Loneliness: A sourcebook of current theory, research and therapy, 206-223, Wiley.

RUSSELL, D.W. et al. (1997): “Loneliness and nursing home admission among rural older adults", Psychology and Aging, 12, 4, 574-589.

SAGAN, O. y MILLER, E. (2017): Narratives of Loneliness. Multidisciplinary Perspectives from the 21st Century, Routledge.

SÁNCHEZ-RODRÍGUEZ, M.M. (2009): Determinantes sociales de la soledad en las personas mayores españolas: Dary recibir apoyo en el proceso de envejecer, Tesis Doctoral, Universidad de Salamanca. 
SÁNCHEZ-RODRÍGUEZ, M.M. et al. (2012): "Loneliness and the exchange of social support among older adults in Spain and the Netherlands", Ageing and Society, 1-25.

SCHWARZBACH, M. et al. (2014): "Social relations and depression in late life: A systematic review", International Journal of Geriatric Psychiatry, 29, 1-21.

SHIOVITZ-EZRA, S. y AYALON, L. (2010): "Situational versus chronic loneliness as risk factors for all-cause mortality”, International Psychogeriatrics, 22 (3), $455-462$

SLETTEB $\emptyset$, $\AA$. (2008): “Safe, but lonely: Living in a nursing home", Vård I Norden, 87, 28, 1, 22-25.

STEIN, J. y TUVAL-MASHIACH, R. (2015): “The social construction of loneliness: An integrative conceptualization", Journal of Constructivist Psychology, 28, 3, 210-227.

Steptoe, A. et al (2004): Loneliness and neuroendocrine, cardiovascular, and inflammatory stress responses in middle-aged men and women. Psychoneuroendocrinology. 29, 593-611, ['https://doi.org/10.1016/So3064530(03)00086-6>].

STEPTOE, A. et al. (2013): "Social isolation, loneliness, and all-cause mortality in older men and women", Proceedings of the National Academy of Sciences of the United States of America, 110 (15), 5797-5801.

STOJANOVIC, J. et al. (2016): “Targeting loneliness and social isolation among the elderly: An update Systematic Review", European Journal of Public Health, 26, 10.

SUNDSTRÖM, G. et al. (2009): “Loneliness among older Europeans”, European Journal of Ageing, 6 (4), 267-75.

SWINDELLS, R. et al. (2013): "Eudaimonic well-being and community arts participation", Perspectives in Public Health, 133 (1), 60-65.

SØNDERBY, L. (2013): “Loneliness: an integrative approach”, Journal of Integrated Social Sciences, 3, 1, 1-29.

TESCH-RÖMER, C. et al. (2013): "Einsamkeittrends in der zweiten Lebenshälfte”, Zeitschrift für Gerontologie und Geriatrie, 46 (3), 237-241.

TSE, M.M.Y. et al. (2014): “The efectiveness of physical exercice training in pain, mobility, and psychological well-being of older persons living in nursing homes", Pain Management Nursing, $15,778-788$.

TYLOVA, V.; PTÁCEK, R. y KUŽELOVÁ, H. (2013): “Emotional and social loneliness in etiology and therapy of mental illness", European Psychiatry, 28, 1.

UMBERSON, D. y MONTEZ, J.K. (2010): "Social relationships and health", Journal of Health and Social Behavior, 51, 54-66.

VELARDE-MAYOL, C.; FRAGA-GIL, S. y GARCÍA-DE-CECILIA, J. M. (2015): "Validación de la escala de soledad de UCLA y perfil social en la población anciana que vive sola", Semergen [<http://dx.doi. org/10.1016/j.semerg.2015.05.017〉].
VICTOR, C. y SULLIVAN, M.P. (2015): “Loneliness and isolation”, en TWIGG, J. y W. Martin (eds.), Handbook of Cultural Gerontology, Routledge, 252-260.

VICTOR, C.R. et al. (2009): The Social World of Older People, Understanding Loneliness and Social Isolation in Later Life, Open University Press.

VITKUS, J. y Horowitz, L.M. (1987): "Poor social performance of lonely people: Lacking a skill or adopting a role?", Journal of Personality and Social Psychology, 52 (6), 1266-1273.

VRBANAC, Z. et al. (2013): "Animal assisted therapy and perception of loneliness in geriatric nursing home residents", Collegium Antropologicum, 37, 973-976.

WAITE, L.J. y GALLAGHER, M. (2000): The case for marriage, Doubleday.

WEISS, R.S. (1983): Loneliness: the experience of emotional and social isolation, MIT Press.

- (1987): "Reflections on the present state of loneliness research", Journal of Social Behavior and Personality, 2 (2), 1-16.

WEISS, R.S.; BOWLBY, J. y PARKES, C.M. (1973): "The study of loneliness”, en WEISS, R.S. (ed.): Loneliness: The experience of emotional and social isolation, MIT Press, 7-30.

- (1973): “Affectional bonds: Their nature and origin”, en R.S. Weiss (1983): Loneliness: The experience of emotional and social isolation, MIT Press, 38-52.

WILSON, R.S. et al. (2007): "Loneliness and risk of Alzheimer's disease", Archives of General Psychiatry, 64, 234-240.

YANG, K. y VICTOR, C. (2011): "Age and loneliness in 25 European nations", Ageing and Society, 31, 1368-1388.

YANGUAS, J. (2018): Ageing and loneliness, VIU-Valencia International University, Valencia.

YANGUAS, J. et al. (en prensa): Vivir es descubrirme, Programa de Mayores, Obra Social "la Caixa".

YANGUAS, J.; PINAZO, S. y TARAZONA, J.(2018): “The complexity of loneliness", Acta Biomed 2018, 89, 2 [rhttp://doi.org/10.23750/abm. v89i2.7404'].

ZEBHAUSER A., et al. (2014): "How much does it hurt to be lonely? Mental and physical differences between older men and women in the KORAAge Study", International journal of geriatric psychiatry, 29 (3), 245-252.

ZHONG, B.L. et al. (2017): "Loneliness and Cognitive Function in Older Adults: Findings From the Chinese Longitudinal Healthy Longevity Survey", The Journals of Gerontology Series B: Psychological Sciences and Social Sciences, 72(1), 120-128 [<http://doi. org/10.1093/geronb/gbw037>]. 